PROCEEDINGS OF THE

AMERICAN MATHEMATICAL SOCIETY

Volume 131, Number 9, Pages 2657-2662

S 0002-9939(03)06831-X

Article electronically published on February 6, 2003

\title{
ON STABLE EQUIVALENCES OF MORITA TYPE FOR FINITE DIMENSIONAL ALGEBRAS
}

\author{
YUMING LIU
}

(Communicated by Martin Lorenz)

\begin{abstract}
In this paper, we assume that algebras are finite dimensional algebras with 1 over a fixed field $k$ and modules over an algebra are finitely generated left unitary modules. Let $A$ and $B$ be two algebras (where $k$ is a splitting field for $A$ and $B$ ) with no semisimple summands. If two bimodules ${ }_{A} M_{B}$ and ${ }_{B} N_{A}$ induce a stable equivalence of Morita type between $A$ and $B$, and if $N \otimes_{A}-$ maps any simple $A$-module to a simple $B$-module, then $N \otimes_{A}-$ is a Morita equivalence. This conclusion generalizes Linckelmann's result for selfinjective algebras. Our proof here is based on the construction of almost split sequences.
\end{abstract}

\section{INTRODUCTION}

Given two finite dimensional $k$-algebras $A$ and $B$, suppose that $M$ is an $A$ - $B$ bimodule and $N$ is a $B$-A-bimodule. Following [2] we say that $M$ and $N$ induce a stable equivalence of Morita type between $A$ and $B$ if $M$ and $N$ are projective both as left and right modules, and if

$$
M \otimes_{B} N \cong A \oplus P
$$

as $A$ - $A$-bimodules, where $P$ is a projective $A$ - $A$-bimodule, and

$$
N \otimes_{A} M \cong B \oplus Q
$$

as $B$ - $B$-bimodules, where $Q$ is a projective $B$ - $B$-bimodule.

Since the projective $A$ - $A$-bimodule $P$ tensoring any $A$-module is a projective $A$-module and since the projective $B$ - $B$-bimodule $Q$ tensoring any $B$-module is a projective $B$-module (see Lemma 2.1), the functor $N \otimes_{A}-$ (defined by the above bimodule $N$ ) induces a stable equivalence between the stable categories $\bmod A$ and $\underline{\bmod } B$, and $M \otimes_{B}-$ induces its quasi-inverse. Note that a stable equivalence $\alpha: \underline{\bmod } A \longrightarrow \underline{\bmod } B$ gives a one-to-one correspondence between the isomorphism classes of indecomposable non-projective modules in $\bmod A$ and $\bmod B$ (see [1, Proposition 1.1, p. 336]).

Important examples of stable equivalences of Morita type are selfinjective algebras which are derived equivalent [7, Corollary 5.5]. Linckelmann in [5] proved that, for two selfinjective algebras $A$ and $B$ having no simple projective modules, if there is an exact functor $F$ which induces a stable equivalence between $A$ and

Received by the editors January 11, 2002 and, in revised form, April 3, 2002.

2000 Mathematics Subject Classification. Primary 16D20; Secondary 16G20.

(C)2003 American Mathematical Society 
$B$ (in fact, $F$ induces a stable equivalence of Morita type by Richard $[8$, Theorem 3.2]), and if $F$ maps any simple $A$-module to a simple $B$-module, then $F$ is a Morita equivalence. Using this result, Linckelmann classified stable equivalences of Morita type between $p$-groups over a complete discrete valuation ring and proved that a stable equivalence of Morita type between the group algebras of two $p$-groups forces these $p$-groups to be isomorphic (see [5], [6]). Recently, Xi studied the representation dimension in [9] and proved in [10] that two finite dimensional algebras which are stably equivalent of Morita type have the same representation dimension. This implies that stable equivalences of Morita type between finite dimensional algebras preserve some nice properties. In this paper, we shall generalize Linckelmann's result ([5 Proposition 2.5]) to arbitrary finite dimensional algebras with no semisimple summands. Namely, we obtain the following theorem.

Theorem 1.1. Let $A$ and $B$ be finite dimensional $k$-algebras (where $k$ is a splitting field for $A$ and $B$ ) with no semisimple summands. If two bimodules ${ }_{A} M_{B}$ and ${ }_{B} N_{A}$ induce a stable equivalence of Morita type between $A$ and $B$, and if $N \otimes_{A}-$ maps any simple $A$-module to a simple $B$-module, then $N \otimes_{A}-$ is a Morita equivalence.

\section{Preliminaries}

For an algebra $A$, we denote by $\bmod A$ and by $\underline{\bmod } A$ the category of finitely generated left $A$-modules and its stable category, respectively. Note that $\bmod A$ is a Krull-Schmidt category. For $X$ in $\bmod A$, we define the top of $X$ by $\operatorname{top}(X)=$ $X / \operatorname{rad}(X)$, where $\operatorname{rad}(X)$ is the radical of $X$. Recall that an $A$-module $X$ is called a generator if the regular module ${ }_{A} A$ is a direct summand of a finite direct sum of $X$.

Suppose that two algebras $A$ and $B$ are stably equivalent of Morita type. We can define functors $T_{M}: \bmod B \longrightarrow \bmod A$ by $X \longmapsto M \otimes_{B} X$ and $T_{N}: \bmod A \longrightarrow$ $\bmod B$ by $Y \longmapsto N \otimes_{A} Y$. Similarly, we have the functors $T_{P}$ and $T_{Q}$. Related to these functors, we have the following two lemmas.

Lemma 2.1 (see [10] Theorem 4.1]). (1) $T_{M}, T_{N}, T_{P}$ and $T_{Q}$ are exact functors.

(2) $T_{M} \circ T_{N} \longrightarrow i d_{\text {modA }} \oplus T_{P}$ and $T_{N} \circ T_{M} \longrightarrow i d_{\bmod B} \oplus T_{Q}$ are natural isomorphisms.

(3) The images of $T_{P}$ and $T_{Q}$ consist of projective modules.

Lemma 2.2. The bimodules ${ }_{A} M_{B}$ and ${ }_{B} N_{A}$ are projective generators both as left and right modules. Therefore $T_{M}$ and $T_{N}$ are faithful functors.

Proof. We prove that ${ }_{A} M$ is a generator. Since ${ }_{B} N$ is projective, there is a natural number $n$ such that ${ }_{B} N$ is a direct summand of ${ }_{B} B^{n}$. It follows that ${ }_{A} M \otimes_{B} N \cong_{A}$ $(A \oplus P)$ is a direct summand of ${ }_{A} M \otimes_{B} B^{n} \cong_{A} M^{n}$. Therefore ${ }_{A} A$ is a direct summand of ${ }_{A} M^{n}$; this implies that ${ }_{A} M$ is a generator in $\bmod A$.

For an $A$-module $X$, we have a unique (up to isomorphism) decomposition $X=$ $X_{1} \oplus X^{\prime}$, where $X_{1}$ has no nonzero projective summands and $X^{\prime}$ is projective. We call $X_{1}$ the non-projective part of $X$. The following lemma is obvious.

Lemma 2.3. If $0 \longrightarrow X \stackrel{f}{\longrightarrow} Y \stackrel{g}{\longrightarrow} Z \longrightarrow 0$ is exact in modA, then there is an exact sequence

$$
0 \longrightarrow X \stackrel{f_{1}}{\longrightarrow} Y_{0} \stackrel{g_{1}}{\longrightarrow} Z_{1} \longrightarrow 0
$$


where $Z \cong Z_{1} \oplus Z^{\prime}, Y \cong Y_{0} \oplus Z^{\prime}, f=\left[\begin{array}{c}f_{1} \\ 0\end{array}\right], g=\left[\begin{array}{cc}g_{1} & g_{2} \\ 0 & g_{3}\end{array}\right]$, and $g_{3}$ is an isomorphism. Moreover, $g$ is a split epimorphism if and only if $g_{1}$ is a split epimorphism.

Lemma 2.4. Let $0 \longrightarrow X \stackrel{i}{\longrightarrow} Y \longrightarrow Z \longrightarrow 0$ be an exact sequence in $\operatorname{modA}$. If $X$ is a simple module and $Z$ has simple top, then either $Y$ has simple top or $Y \cong X \oplus Z$.

Proof. If $i(X)$ lies in $\operatorname{rad}(Y)$, then $\operatorname{top}(Y)=\operatorname{top}(Z)$; therefore $Y$ has simple top.

If $i(X)$ is not in $\operatorname{rad}(Y)$, then $i$ induces an isomorphism $X \longrightarrow S$ which we also denote by $i$, where $S$ is a direct summand of $\operatorname{top}(Y)$. We define $j: Y \longrightarrow X$ to be the composition $Y \stackrel{\rho}{\longrightarrow} S \stackrel{i^{-1}}{\longrightarrow} X$ where $\rho$ is the canonical projection. Then we have $j \circ i=i d_{X}$. This implies that $i$ is split, and therefore $Y \cong X \oplus Z$.

Let $A$ be a finite dimensional algebra. Suppose that $\left\{S_{1}, \ldots, S_{n}\right\}$ is a complete set of non-isomorphic simple $A$-modules and $\left\{P_{1}, \ldots, P_{n}\right\}$ is the corresponding projective covers. Recall that the Cartan matrix $C(A)$ of the algebra $A$ is an $n \times n$ matrix, with its $i$-j-entry given by the number of composition factors of $P_{j}$ which are isomorphic to $S_{i}$.

The following lemma is well-known (see, for example, [3] §54.16]).

Lemma 2.5. Let $A$ be a finite dimensional $k$-algebra where $k$ is a splitting field for $A$, and let $X$ be an $A$-module. Then for any $1 \leq i \leq n$, the number of composition factors of $X$ which are isomorphic to $S_{i}$ is $\operatorname{dim}_{k} \operatorname{Hom}\left(P_{i}, X\right)$.

Remark. $k$ is a splitting field for $k$-algebra $A$ if and only if every $A$-endomorphism ring of simple $A$-module is isomorphic to the base field $k$. In particular, any algebraically closed field $k$ is a splitting field for algebras over $k$ (see [3, $\S 29]$ ).

\section{Proof of Theorem 1.1}

In this section, we shall give a proof of the generalization of Linckelmann's theorem. Our proof here is based on the construction of almost split sequences. For the basic theory of almost split sequences, we refer to [1].

Proposition 3.1. Let $A$ and $B$ be finite dimensional $k$-algebras with no semisimple summands, and let $\left\{S_{1}, \ldots, S_{n}\right\}$ be a complete set of non-isomorphic simple Amodules and $\left\{P_{1}, \ldots, P_{n}\right\}$ be the corresponding projective covers. If two bimodules ${ }_{A} M_{B}$ and ${ }_{B} N_{A}$ induce a stable equivalence of Morita type between $A$ and $B$, and if $N \otimes_{A}-$ maps any simple $A$-module to a simple $B$-module, then we have the following:

(1) $\left\{T_{N}\left(S_{i}\right) \mid i=1, \ldots, n\right\}$ is a complete set of non-isomorphic simple B-modules;

(2) $\left\{T_{N}\left(P_{i}\right) \mid i=1, \ldots, n\right\}$ is a complete set of non-isomorphic indecomposable projective $B$-modules.

Proof. (1) First, we show that every projective $B$-module $E$ lies in

$$
\operatorname{add}\left(\bigoplus_{i=1}^{n} T_{N}\left(P_{i}\right)\right)
$$

Since $T_{M}(E)$ is a projective $A$-module, we have $T_{M}(E) \in \operatorname{add}\left(\bigoplus_{i=1}^{n} P_{i}\right)$. It follows from $T_{N} \circ T_{M}(E) \cong E \oplus T_{Q}(E) \in \operatorname{add}\left(\bigoplus_{i=1}^{n} T_{N}\left(P_{i}\right)\right)$ that $E$ lies in $\operatorname{add}\left(\bigoplus_{i=1}^{n} T_{N}\left(P_{i}\right)\right)$. Since all composition factors of $T_{N}\left(P_{i}\right)(1 \leq i \leq n)$ occur in $\left\{T_{N}\left(S_{i}\right) \mid i=1, \ldots, n\right\}$, 
we know that $\left\{T_{N}\left(S_{i}\right) \mid i=1, \ldots, n\right\}$ contains all isomorphism classes of simple $B$ modules. To finish the proof we need to show that $T_{N}\left(S_{i}\right) \neq T_{N}\left(S_{j}\right)$ for all $i \neq j$. It suffices to prove this when $S_{i}$ and $S_{j}$ are projective modules. Note that $S_{i}$ and $S_{j}$ are non-injective modules since $A$ has no semisimple summands. By [1, Proposition 2.6, p.151], we have an almost split sequence $0 \longrightarrow S_{i} \stackrel{f}{\longrightarrow} T_{i} \stackrel{g}{\longrightarrow} \operatorname{Tr} D S_{i} \longrightarrow 0$, where $T_{i}$ is projective. Applying $T_{N}$ we get an exact sequence $0 \longrightarrow T_{N}\left(S_{i}\right) \stackrel{T_{N}(f)}{\longrightarrow}$ $T_{N}\left(T_{i}\right) \stackrel{T_{N}(g)}{\longrightarrow} T_{N}\left(\operatorname{Tr} D S_{i}\right) \longrightarrow 0$ in $\bmod B$. By Lemma 2.3 , we get an exact sequence

$$
0 \longrightarrow T_{N}\left(S_{i}\right) \stackrel{T_{N}(f)_{1}}{\longrightarrow} T_{N}\left(T_{i}\right)_{0} \stackrel{\left.T_{N}(g)\right)_{1}}{\longrightarrow} T_{N}\left(\operatorname{Tr} D S_{i}\right)_{1} \longrightarrow 0,
$$

where $T_{N}\left(\operatorname{Tr} D S_{i}\right)_{1}$ is the non-projective part of $T_{N}\left(\operatorname{Tr} D S_{i}\right), T_{N}\left(\operatorname{Tr} D S_{i}\right)=$ $T_{N}\left(\operatorname{Tr} D S_{i}\right)_{1} \oplus T_{N}\left(\operatorname{Tr} D S_{i}\right)^{\prime}, T_{N}\left(T_{i}\right) \cong T_{N}\left(T_{i}\right)_{0} \oplus T_{N}\left(\operatorname{Tr} D S_{i}\right)^{\prime}, T_{N}(f)=\left[\begin{array}{c}T_{N}(f)_{1} \\ 0\end{array}\right]$, and $T_{N}(g)=\left[\begin{array}{cc}T_{N}(g)_{1} & T_{N}(g)_{2} \\ 0 & T_{N}(g)_{3}\end{array}\right]$. Since $T_{N}\left(S_{i}\right)$ is simple, $T_{N}(g)_{1}$ is a projective cover. We want to show that $(*)$ is an almost split sequence.

Clearly, there is an almost split sequence

$$
0 \longrightarrow X \longrightarrow E \stackrel{h}{\longrightarrow} T_{N}\left(\operatorname{Tr} D S_{i}\right)_{1} \longrightarrow 0 .
$$

We claim that $E$ is projective. Assume that $E$ is not projective. Write $E=E_{1} \oplus E^{\prime}$, where $E_{1} \neq 0$ is the non-projective part of $E$. Since $T_{N}\left(\operatorname{Tr} D S_{i}\right)_{1}$ is indecomposable non-projective, and since $E=E_{1} \oplus E^{\prime} \longrightarrow T_{N}\left(\operatorname{Tr} D S_{i}\right)_{1}$ is a minimal right almost split morphism, there exists a morphism $F \longrightarrow T_{M}\left(T_{N}\left(\operatorname{Tr} D S_{i}\right)_{1}\right)_{1}$ with $F$ projective in $\bmod A$ such that $T_{M}\left(E_{1}\right)_{1} \oplus F \longrightarrow \operatorname{Tr} D S_{i}$ is a minimal right almost split morphism ([1, Proposition 1.3, p.337]), where $T_{M}\left(E_{1}\right)_{1} \neq 0$ is the non-projective part of $T_{M}\left(E_{1}\right)$, and $T_{M}\left(T_{N}\left(\operatorname{Tr} D S_{i}\right)_{1}\right)_{1} \cong \operatorname{Tr} D S_{i}$ is the non-projective part of $T_{M}\left(T_{N}\left(\operatorname{Tr} D S_{i}\right)_{1}\right)$. But $T_{i} \longrightarrow \operatorname{Tr} D S_{i}$ is a minimal right almost split morphism with $T_{i}$ projective; this contradicts the uniqueness of minimal right almost split morphism! Since $E$ must be projective, it follows from [1, Theorem 3.3, p.154] that $h$ is a projective cover. Hence the exact sequences $(*)$ and $(* *)$ are isomorphic. This implies that $(*)$ is also an almost split sequence.

Similarly, we have an almost split sequence

$$
0 \longrightarrow T_{N}\left(S_{j}\right) \longrightarrow T_{N}\left(T_{j}\right)_{0} \longrightarrow T_{N}\left(\operatorname{Tr} D S_{j}\right)_{1} \longrightarrow 0,
$$

where $T_{N}\left(\operatorname{Tr} D S_{j}\right)_{1}$ is the non-projective part of $T_{N}\left(\operatorname{Tr} D S_{j}\right)$. Suppose that $T_{N}\left(S_{i}\right)$ $\cong T_{N}\left(S_{j}\right)$. Then $T_{N}\left(\operatorname{Tr} D S_{i}\right)_{1} \cong T_{N}\left(\operatorname{Tr} D S_{j}\right)_{1}$ by the basic properties of almost split sequences. But $\operatorname{Tr} D S_{i}$ and $\operatorname{Tr} D S_{j}$ are non-isomorphic, indecomposable nonprojective modules. This contradicts the fact that $T_{N}$ induces a stable equivalence, and therefore (1) follows.

(2) By the previous proof, we only need to show that $T_{N}\left(P_{i}\right)$ is indecomposable for all $1 \leq i \leq n$. In fact, we shall prove the following more general result: if an $A$-module $X$ has simple top, then the $B$-module $T_{N}(X)$ also has simple top. We prove this by induction on the length of $X$. For $l(X)=1$, the module $X$ is simple, and $T_{N}(X)$ is simple by assumption. For $l(X)=m>1$, take an exact sequence $0 \longrightarrow S \longrightarrow X \longrightarrow X / S \longrightarrow 0$, where $S$ is a simple submodule of $X$ and therefore $X / S$ has simple top. Applying $T_{N}$ we get an exact sequence $0 \longrightarrow$ $T_{N}(S) \longrightarrow T_{N}(X) \longrightarrow T_{N}(X / S) \longrightarrow 0$, where $T_{N}(S)$ is simple, and $T_{N}(X / S)$ has simple top by induction. By Lemma 2.4, either $T_{N}(X)$ has simple top or $T_{N}(X) \cong T_{N}(S) \oplus T_{N}(X / S)$. Suppose that $T_{N}(X) \cong T_{N}(S) \oplus T_{N}(X / S)$. Applying 
$T_{M}$ we have $X \cong S \oplus X / S$ since $X / S$ is an indecomposable non-projective module. This contradicts the indecomposability of $X$, and completes our proof.

Proof of Theorem 1.1. By Lemma 2.2, $T_{N}$ is a faithful functor and induces a monomorphism between algebras: $\operatorname{End}_{A}(A) \longrightarrow \operatorname{End}_{B}\left(T_{N}(A)\right)$. By Proposition 3.1, $T_{N}$ induces a bijection between the sets of isomorphism classes of indecomposable projective modules over $A$ and $B$. On the other hand, $T_{N}$ is an exact functor which gives a one-to-one correspondence between the sets of isomorphism classes of simple modules over $A$ and $B$. It follows that $T_{N}$ preserves the Cartan matrix. By Lemma 2.5, Cartan matrix is given by $k$-dimensions of homomorphism spaces between indecomposable projective modules. Therefore we have

$$
\operatorname{dim}_{k} \operatorname{Hom}_{A}\left(P_{i}, P_{j}\right)=\operatorname{dim}_{k} \operatorname{Hom}_{B}\left(T_{N}\left(P_{i}\right), T_{N}\left(P_{j}\right)\right)
$$

for all $1 \leq i, j \leq n$. Assume that $A \cong \bigoplus_{i=1}^{n} P_{i}^{m_{i}}$. Then we have

$$
\operatorname{dim}_{k} \operatorname{End}_{A}(A)=\sum_{i, j=1}^{n} \operatorname{dim}_{k} \operatorname{Hom}_{A}\left(P_{i}, P_{j}\right) m_{i} m_{j}
$$

and

$$
\operatorname{dim}_{k} \operatorname{End}_{B}\left(T_{N}(A)\right)=\sum_{i, j=1}^{n} \operatorname{dim}_{k} \operatorname{Hom}_{B}\left(T_{N}\left(P_{i}\right), T_{N}\left(P_{j}\right)\right) m_{i} m_{j} .
$$

Thus $\operatorname{End}_{A}(A) \longrightarrow \operatorname{End}_{B}\left(T_{N}(A)\right)$ is an isomorphism. Since $A \cong \operatorname{End}_{A}(A)^{o p}$ and $T_{N}(A) \cong N$ is a projective generator as $B$-module, we know that $A \cong \operatorname{End}_{B}(N)^{o p}$. Hence $T_{N}$ is a Morita equivalence between $A$ and $B$ by the Morita theorem (see, for example, [4, Theorem 8.4.5]).

Remark. If $k$ is a perfect field, then by [3, §54.19] we know that the condition $k$ is a splitting field for $A$ and $B$ can be weakened as follows: $\operatorname{dim}_{k} E n d_{A}(S)=$ $\operatorname{dim}_{k} \operatorname{End}_{B}\left(T_{N}(S)\right)$ for any simple $A$-module $S$.

\section{ACKNOWLEDGEMENTS}

I thank Changchang Xi for his encouragement and many helpful suggestions. I also thank Markus Linckelmann for some correspondence on his paper [5]. This research work is supported by the Doctoral Program Foundation of the Education Ministry of China (No. 20010027015).

\section{REFERENCES}

[1] M. Auslander, I. Reiten and S. O. Smalø, Representation theory of Artin algebras, Cambridge University Press, 1995. MR 96c:16015

[2] M. Broué, Equivalences of blocks of group algebras, Finite dimensional algebras and related topics, V. Dlab and L. L. Scott (eds.), Kluwer, 1994, 1-26. MR 97c:20004

[3] C. W. Curtis and I. Reiner, Representation theory of finite groups and associative algebras, Wiley-Interscience, New York, 1962. MR 26:2519, reprint MR 90g:16001

[4] Y. A. Drozd and V. V. Kirichenko, Finite dimensional algebras, Springer-Verlag, Berlin, 1994. MR 95i:16001

[5] M. Linckelmann, Stable equivalences of Morita type for selfinjective algebras and $p$-groups, Math. Zeit. 223(1996), 87-100.

[6] M. Linckelmann, On stable equivalences of Morita type, LNM 1685, Derived equivalences for group rings, 1998, 221-232.

[7] J. Rickard, Derived equivalences as derived functors, J. London Math. Soc. 43(1991), 37-48. MR 92b:16043 
[8] J. Rickard, Some recent advances in modular representation theory, CMS Conf. Proc. 23(1998), 157-178. MR 99h:20011

[9] C. C. Xi, On the representation dimension of finite dimensional algebras, J. of Algebra 226, no. 1(2000), 332-346. MR 2001d:16027

[10] C. C. Xi, Representation dimension and quasi-hereditary algebras, Adv. in Math. 168(2002), 193-212. CMP 2002:15

Department of Mathematics, Beijing Normal University, 100875 Beijing, People's Republic of China

E-mail address: liuym2@263.net 